\title{
A novel 8-bp duplication in ADAT3 causes mild intellectual disability
}

\author{
Ahmad Reza Salehi Chaleshtori ${ }^{1}$, Noriko Miyake ${ }^{2}$, Mohammad Ahmadvand ${ }^{3}$, Oranous Bashti ${ }^{4}$, \\ Naomichi Matsumoto ${ }^{2}$ and Mehrdad Noruzinia'
}

\begin{abstract}
Inosine is a base located at wobble position 34 of the tRNA anticodon stem-loop, enabling the recognition of more than one codon in the translation process. A heterodimer consists of ADAT3 and ADAT2 and is involved in the adenosine-to-inosine conversion in tRNA. Here, we report the second novel ADAT3 mutation in a patient with microcephaly, intellectual disability, and hyperactivity. These findings constitute a second mutation and expand the clinical spectrum of extremely rare ADAT3 mutations.
\end{abstract}

Adenosine (A)-to-inosine (I) RNA editing is a posttranscriptional RNA process capable of generating RNA and protein diversity ${ }^{1}$. Inosine at wobble position 34 of tRNA anticodons can translate codons ending in uracil, cytosine, or adenine ${ }^{2}$. The modification, which creates an I from an A at position 34 (wobble position) of tRNA, is catalyzed by the heterodimeric enzyme, adenosine deaminase, tRNA-specific 3 (ADAT3)/ADAT2 ${ }^{2}$.

Alazami et al. described a homozygous ADAT3 mutation (c.382 G > A, p.Val128Met) in 24 affected individuals with autosomal-recessive mental retardation 36 (MRT36; MIM*615286) from eight consanguineous Arab families ${ }^{3}$. Very recently, El-Hattab et al. reported an additional 15 patients with an identical homozygous ADAT3 mutation in 15 affected individuals from 11 Arab families. In the previous reports, strabismus, microcephaly, failure to thrive, and abnormal brain structure were frequently seen in such patients.

We encountered a 6-year-old female presenting with intellectual disability, mild cognitive impairment, attention deficit, hyperactivity disorder, neurodevelopmental delay,

Correspondence: Mehrdad Noruzinia (noruzinia@modares.ac.ir)

'Department of Medical Genetics, Faculty of Medical Sciences, Tarbiat Modares University, P.O. Box 14115-331 Tehran, Iran

2Department of Human Genetics, Yokohama City University Graduate School of Medicine, Yokohama 236-0004, Japan

Full list of author information is available at the end of the article speech delay, and microcephaly. The patient's face was asymmetric, and her nasal bridge was depressed. She was born to healthy Iranian consanguineous parents (Fig. 1a). The proband visited our genetic center seeking a genetic testing service. Considering the clinical findings, the targeted sequencing of 12 genes associated with microcephaly (SLC25A19, STIL, ASPM, CEP135, MCPH1, CDK5RAP2, CENPJ, CEP152, WDR62, ZNF335, ADAT3, and EFTUD2) was provided. After obtaining informed consent, genomic DNA of peripheral blood leukocytes was extracted and used for the genome partitioning. Targeted capture was performed using the GeneRead DNAseq Custom Panel V2 (QIAGEN, Hilden, Germany), and the libraries were sequenced to mean $>80-100 \times$ coverage on a HiSeq2000 sequencing platform (Illumina, San Diego, CA, USA). For read mapping and variant analysis, sample sequences were aligned to the human reference genome (GRCh37/hg19) using Burrows-Wheeler Aligner ${ }^{4}$. To identify variants relevant to the disease, the obtained data were manipulated using picard and processed with the Genome Analysis Toolkit (GATK refv1.2905) .

Through our targeted sequencing, we identified a homozygous 8-bp duplication in ADAT3 (c.99_106dupGAGCCCGG, p.(Glu36Glyfs"44); Fig. 1b). This variant was not previously registered in the 1000 genomes database (http://browser.1000genomes.org/index.html), ExAC browser (http://exac.broadinstitute.org/), or EVS 
A

I

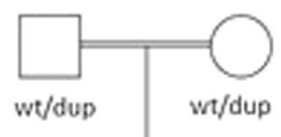

II

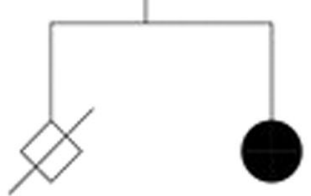

Patient

(c.99_106dupGAGCCCGG)

Wild-type Allele

B

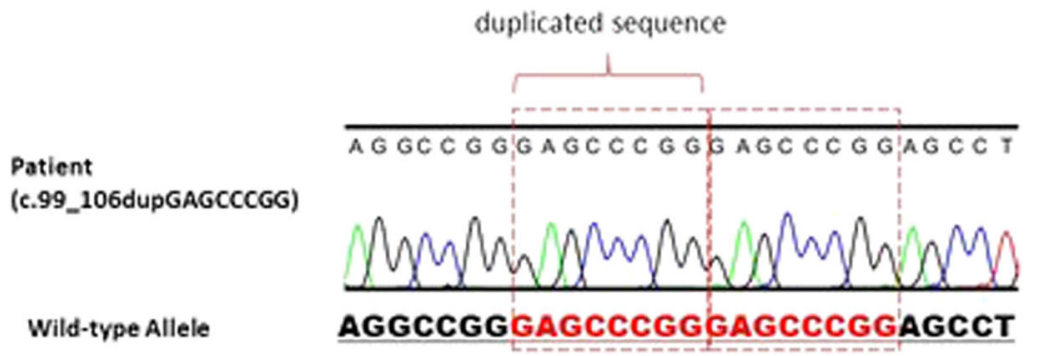

Fig. 1 Segregation status of the mutation and Sanger confirmation of c.99_106dupGAGCCCGG mutation in the proband a Pedigree information and segregation status of the ADAT3 8-bp duplication. b Chromatogram of the c.99_106dupGAGCCCGG, p.(Glu36Glyfs*44) mutation

Table 1 Clinical features of the patient compared to previous report of $A D A T 3$-related cognitive impairment

\begin{tabular}{ll}
\hline This report & Previous report \\
\hline Cognition & \\
Intellectual disability & Intellectual disability \\
Mild to moderate cognitive & Moderate to severe cognitive \\
impairment & impairment \\
Attention deficit hyperactivity disorder & Aggressive/hyperactivity \\
(ADHD) & \\
Development & \\
Neurodevelopmental delay & Developmental delay \\
Speech delay & No speech ability \\
Face-skull & \\
Microcephaly & Microcephaly \\
Asymmetric face & Elongated face with prominent \\
& nose \\
Depressed nasal bridge & Depressed nasal bridge \\
No strabismus & Strabismus \\
\hline
\end{tabular}

(http://evs.gs.washington.edu/EVS/). Since this gene has one coding exon, the frameshift mutation might produce a truncated protein. We confirmed both parents as heterozygous carriers (Fig. 1b), agreeing with the autosomalrecessive mode of inheritance.

The proband we present here shared many clinical features with the patients reported by El-Hattab and Alazami $^{3}$, including hyperactivity, developmental delay, microcephaly, depressed nasal bridge, and asymmetric face, which were commonly seen in the current patient. In contrast, our patient showed speech delay, while ElHattab reported speech incapability (no words) in patients with the c.382 G > A mutation. Most patients with the c.382 G > A mutation in ADAT3 have been characterized with moderate to severe cognitive impairment ${ }^{3,6}$, while the present patient was a sufferer from mild intellectual disability. Moreover, previous reports on ADAT3 mutation noted strabismus as an accompanying sign of cognitive impairment in patients with the c.382G > A mutation $^{3,6}$; however, this patient did not show strabismus. Other clinical findings were consistent with previous reports ${ }^{3,6}$ (Table 1); therefore, the difference in clinical features might be due to the different mutational effects of respective mutations.

In conclusion, we report a novel and second ADAT3 mutation in a patient with intellectual disability and propose that $A D A T 3$ sequencing should be considered for intellectual disability in the Middle East.

\section{HGV Database}

The relevant data from this Data Report are hosted at the Human Genome Variation Database at https://doi.org/10.6084/m9.figshare.hgv.1942.

\section{Acknowledgements}

We would like to thank the patient and her family members for participating in this study.

\section{Author details}

${ }^{1}$ Department of Medical Genetics, Faculty of Medical Sciences, Tarbiat Modares University, P.O. Box 14115-331 Tehran, Iran. ${ }^{2}$ Department of Human Genetics, Yokohama City University Graduate School of Medicine, Yokohama 236-0004, Japan. ${ }^{3}$ Hematology, Oncology and Stem Cell Transplantation Research Center, Shariati Hospital, Tehran University of Medical Sciences, Tehran P.O. Box 14114, Iran. ${ }^{4}$ Laboratory of Medical Genetics, iHealth Clinics, P.O. Box 1913874416

Tehran, Iran

Conflict of interest

The authors declare that they have no conflict of interest.

\section{Publisher's note}

Springer Nature remains neutral with regard to jurisdictional claims in published maps and institutional affiliations.

Received: 19 December 2017 Revised: 10 March 2018 Accepted: 11 March 2018.

Published online: 21 May 2018 


\section{References}

1. Tang, W., Fei, Y. \& Page, M. Biological significance of RNA editing in cells. Mol. Biotechnol. 52, 91-100 (2012).

2. Gerber, A. P. \& Keller, W. An adenosine deaminase that generates inosine at the wobble position of tRNAs. Science 286, 1146-1149 (1999).

3. Alazami, A. M. et al. Mutation in ADAT3, encoding adenosine deaminase acting on transfer RNA, causes intellectual disability and strabismus. J. Med. Genet. 50, 425-430 (2013).
4. Li, H. \& Durbin, R. Fast and accurate short read alignment with Burrows-Wheeler transform. Bioinformatics 25, 1754-1760 (2009).

5. DePristo, M. A. et al. A framework for variation discovery and genotyping using next-generation DNA sequencing data. Nat. Genet. 43, 491-498 (2011).

6. El-Hattab, A. W. et al. ADAT3-related intellectual disability: further delineation of the phenotype. Am. J. Med. Genet. A 170a, 1142-1147 (2016). 